\title{
Commentary: Quantitative home-based assessment of Parkinson's symptoms: The SENSE-PARK feasibility and usability study
}

\author{
Godinho $\mathrm{C}^{1,2,3}$, Domingos J1,3, Ferreira JJ',3,4* \\ 'Instituto de Medicina Molecular, Faculty of Medicine, University of Lisbon, Portugal \\ ${ }^{2}$ Center for Interdisciplinary Research Egas Moniz (CiiEM), Instituto Superior de Ciências da Saúde Egas Moniz, Monte de Caparica, Portugal \\ ${ }^{3}$ CNS-Campus Neurológico Sénior, Torres Vedras, Portugal \\ ${ }^{4}$ Laboratory of Clinical Pharmacology and Therapeutics, Faculty of Medicine, University of Lisbon, Lisbon, Portugal
}

\section{Article Info}

\section{Article Notes}

Received: 04/30/2016

Accepted: 05/16/2016

\section{Correspondence:}

Laboratory of Clinical Pharmacology and Therapeutics

Faculty of Medicine, University of Lisbon

Av. Prof. Egas Moniz 1649-028 Lisboa, Portugal. Telephone: 217802120

Fax number: 217802129

Email: joaquimiferreira@gmail.com

(c) 2016 Ferreira JJ. This article is distributed under the terms of the Creative Commons Attribution 4.0 International License.

\section{ABSTRACT}

Healthcare professionals and pharmaceutical companies invest a great amount of time and effort in continuously creating electronic health solutions. These technology system developments may represent a step forward in care as ultimately it is not possible to manage what cannot be evaluated.

Yet, the use of future generations of technology depends on their specific design, fabrication, distribution, and, most importantly, patients adopting these new technologies as life companions. Data management and the use of artificial intelligence appear as new technological challenges. The overload, sharing and handling of information give rise to new legal, social, and ethical discussions in a field where there is a lack of universal criteria for data ownership, privacy and sharing.

Future technological progress requires much cooperation between multidisciplinary teams including sufficient sharing and benchmarking within open access frameworks. 


\section{Commentary}

The revolution of sensors and wearables, the Internet of Things ${ }^{1}$, and the new ways of processing data using thought machine learning and artificial intelligence for instance, are beginning to greatly change healthcare delivery to all patients, but more specifically to those suffering from chronic diseases. Treatment of Parkinson's disease (PD) and other neurodegenerative and progressive disorders are guaranteed to benefit from this innovative and useful approach.

The complex and fluctuating nature of PD makes it difficult to assess in a comprehensive way. Continuous non-obtrusive monitoring during activities of daily living could provide more objective and representative data and improve current care. It may enhance collaboration between PD patients and medical teams through a better understanding of therapy effectiveness and monitoring of disease progression.

The SENSE-PARK project seeks to support and empower PD patients in their home environment using a novel sensory information system that monitors daily-liferelevant parameters of PD and their changes over the time.

\section{Strenghts}

One of the strengths of this study is the detailed and robust approach taken by the authors to validate the system. In fact, there are many different technologies being developed for use in PD, yet, the validity of most of these devices and technologies in PD is still unclear and there are persisting concerns about whether they actually measure what they are intended for $^{2,3}$. This study thus raises a fundamental question regarding the need for an objective validation processes and is an example of how such technology can be tested in a PD-specific context. The determination of repeatability and reliability of new technology assessment methods remains challenging. Whether the existing assessments can ultimately be replaced or complement new technology assessments is also an open and ongoing discussion in the PD health community.

Another strength of the study is that measurement of PD symptoms is performed in a daily living (ecological) setting and not in a clinical environment that may poorly reflect patients' real life situations. More accurate feedback about the well-being of the patient is obtained with simple and frequent momentary measures, reducing bias and error in recollected self-reported data. Additionally, there may be better communication with the medical team and a better adaptation of therapy to the real needs of the patient.

Finally, another important strength of this project is that it has positively impacted the engagement of many different professionals form different fields (neurologist, engineers, physiotherapists, exercise physiologists, researchers, etc.) and more importantly it has had a positive impact, since the very beginning, on the engagement of patients with PD.

\section{Barriers}

\section{Patient Adherence}

However, a few fundamental problems have been evoked in the literature regarding the adherence of patients with PD. Studies have showed that there was a high dropout rate for smartphones, $26 \%$ use it only once and about $70 \%$ of patients used it more than 10 times $^{4}$. Understanding the reason underlying why one system is preferred over another allows us to streamline the use of such systems even further.

In our study we observed that in the context of cognitive domains, these type of instruments can be a "double-edged sword". On the one hand, cognitive impairment, often associated with neurodegenerative diseases, may represent a barrier to a system's use, while on the other hand, it can become an interesting way of cognitive evaluation or training.

In fact, elderly patients with PD may require more training because they are less likely to be familiar with computer systems compared to younger generations. If they are not comfortable in using the system or find it frustrating to use, compliance is unlikely to be good. Yet, being elderly, or unfamiliar with computer technology does not necessarily represent a major problem if systems are well-designed and thought out from the patient's point of view, alongside specific teaching sessions on its use.

Making systems user-friendly and providing direct and continuous feedback about the individual health condition are therefore key issues in PD, and this echoes the results of both this study and others 5 . However, the design and type of sensors deserves further reflection because considerations such as size, visual display, ergonomics and ease to use may also be key factors.

It is vital that systems are simple to understand and easy to operate. The sense-park system includes requirements identified as important for users aged above 65 years: systems should prolong independence, ensure privacy of data, be compact (light and small), have a long battery life and be placed on the wrist using a bracelet instead of directly on the $\operatorname{skin}^{6}$, but some adverse events cannot be avoided completely such as the occurrence of minor skin irritations. Participants also stated that they would prefer sensors that were embedded into clothing (accessories) instead of sensors that had to be worn. There was a common perception that acceptance of a sensor system would increase if they were more unnoticeable. Esthetics still is an important aspect for users ${ }^{6}$.

Given the increasing advances in technology, the use of 
other types of portable technology, such as mobile phones, may further facilitate the collection of data and continuous compliance because people carry their mobile phones with during most of the day.

\section{Data management}

The data transfer aspect is of interest in its own way. Electronic data capture has the potential to significantly reduce the time taken to obtain and manage clinical data. Nevertheless, some questions have arisen in relation to this context: what and how much of the data collected is useful and should be shared with health professionals? Big data versus big enough data, better data, cheaper, cost saver, low burden are issues that deserve further reflection in the coming years.

Ultimately, it is necessary to know how to make sense of the data; what do we want technology to accomplish and what do we want to accomplish in PD? Biomarker identification through sensing and closed loop systems are warranted and are expected to reduce the burden of patient management and may lead to automated programming. The possibility of patients uploading their disease specific profiles and receiving personalized assessment tools and later management tips is an ultimate envision of such use of technology. Because of new challenges such as data privacy and ownership are already at the core of ongoing discussion, a range of ethical, legal and sociological issues need to be considered 1 . Ultimately the ethics applied in using technology differ according to context and population and will require a specific approach. Consensus on data handling might evolve into regularized data depending on their type, usage, and users.

\section{References}

1. Pasluosta CF, Gassner H, Winkler J, Klucken J, Eskofier BM. An Emerging Era in the Management of Parkinson's Disease: Wearable Technologies and the Internet of Things. IEEE J Biomed Health Inform 2015; 19: 1873-1881.

2. Godinho C, Domingos J, Cunha G, Santos AT, Fernandes RM, Abreu D, et al. A systematic review of the characteristics and validity of monitoring technologies to assess Parkinson's disease. J Neuroeng Rehabil. 2016; 13: 24

3. Maetzler W, Domingos J, Srulijes K, Ferreira JJ, Bloem BR. Quantitative wearable sensors for objective assessment of Parkinson's disease. Mov Disord 2013; 28: 1628-1637.

4. Ledger D, McCaffrey D. Inside wearables: how the science of human behavior change offers the secret to long-term engagement, LLC EP ed: Endeavour Partners LLC, 2014.

5. van Uem J, Isaacs T, Lewin A, Bresolin E, Salkovic D, Espay AJ, et al. A Viewpoint on Wearable Technology-Enabled Measurement of Wellbeing and Health-Related Quality of Life in Parkinson's Disease. J Parkinsons Dis. 2016.

6. Bergmann $\mathrm{JH}, \mathrm{McGregor} A \mathrm{H}$. Body-worn sensor design: what do patients and clinicians want? Ann Biomed Eng 2011; 39: 2299-2312. 